\title{
The Impact of Cold Storage on Adenosine Diphosphate-Mediated Platelet Responsiveness
}

\author{
Juergen Koessler ${ }^{1}$ Philipp Klingler ${ }^{1}$ Marius Niklaus $^{1} \quad$ Katja Weber $^{1}$ Angela Koessler ${ }^{1}$ \\ Markus Boeck $^{1}$ Anna Kobsar ${ }^{1}$ \\ ${ }^{1}$ Institute of Clinical Transfusion Medicine and Haemotherapy, \\ University of Wuerzburg, Germany \\ Address for correspondence Juergen Koessler, MD, Institute of \\ Clinical Transfusion Medicine and Haemotherapy, University of \\ Wuerzburg, Oberduerrbacher Straße 6, Wuerzburg D-97080, \\ TH Open 2020;4:e163-e172. \\ Germany (e-mail: koessler_j@ukw.de).
}

\begin{abstract}
Keywords

- platelet physiology

- cold storage

- adenosine diphosphate

- purinergic receptors

- inhibitory signaling

Introduction Cold storage of platelets is considered to contribute to lower risk of bacterial growth and to more efficient hemostatic capacity. For the optimization of storage strategies, it is required to further elucidate the influence of refrigeration on platelet integrity. This study focused on adenosine diphosphate (ADP)-related platelet responsiveness.

Materials and Methods Platelets were prepared from apheresis-derived platelet concentrates or from peripheral whole blood, stored either at room temperature or at $4^{\circ} \mathrm{C}$. ADP-induced aggregation was tested with light transmission. Activation markers, purinergic receptor expression, and $\mathrm{P} 2 \mathrm{Y} 12$ receptor function were determined by flow cytometry. P2Y1 and P2X1 function was assessed by fluorescence assays, cyclic nucleotide concentrations by immunoassays, and vasodilator-stimulated phosphoprotein (VASP)-phosphorylation levels by Western blot analysis.

Results In contrast to room temperature, ADP-induced aggregation was maintained under cold storage for 6 days, associated with elevated activation markers like fibrinogen binding or CD62P expression. Purinergic receptor expression was not essentially different, whereas $\mathrm{P} 2 \mathrm{Y} 1$ function deteriorated rapidly at cold storage, but not P2Y12 activity. Inhibitory pathways of cold-stored platelets were characterized by reduced responses to nitric oxide and prostaglandin E1. Refrigeration of citrated whole blood also led to the attenuation of induced inhibition of platelet aggregation, detectable within 24 hours.

Conclusion ADP responsiveness is preserved under cold storage for 6 days due to stable P2Y12 activity and concomitant disintegration of inhibitory pathways enabling a higher reactivity of stored platelets. The ideal storage time at cold temperature for the highest hemostatic effect of platelets should be evaluated in further studies.
\end{abstract}

\section{Introduction}

Platelet transfusions are required for the prevention and therapy of hemorrhage related to thrombocytopenia or platelet disorders. ${ }^{1}$ In transfusion medicine, it is an important issue to optimize the ex vivo storage conditions for platelet concentrates (PC) to preserve platelet integrity and

received

March 27, 2020 accepted after revision June 9, 2020
DOI https://doi.org/

10.1055/s-0040-1714254. ISSN 2512-9465. to minimize storage lesions. ${ }^{2}$ During the last decades, many amendments have been made by adapting container material, storage media, or technical procedures. Depending on country-specific regulations, PC are commonly stored for 4 to 7 days under continuous agitation at room temperature (RT). ${ }^{3}$ Until the 1980 s, refrigerated PC with storage temperatures of 1 to $6^{\circ} \mathrm{C}$ have also been considered as components
(C) 2020 Georg Thieme Verlag KG Stuttgart . New York
License terms

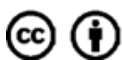


for transfusions. ${ }^{4}$ However, the transfusion of cold platelets was associated with reduced platelet survival compared with room temperature platelets. ${ }^{5}$ In addition, the number of patients with hypoproliferative thrombocytopenia due to hematological malignancies continuously increased requiring long-circulating platelets. In consequence, room temperature was implemented as the standard for the storage of PC, although bearing a higher risk of bacterial growth.,

Recently, the interest in cold platelets has awakened. Due to their higher responsiveness, they are considered to be advantageous for acute hemorrhage, for example, in cardiovascular surgery ${ }^{7,8}$ or in military settings. ${ }^{4}$ Studies with cold platelets showed superior aggregation responses to agonists in vitro. ${ }^{9-11}$ In vivo, cold platelets were able to reduce bleeding more efficiently in individuals under aspi$\operatorname{rin}^{12}$ or in patients with thrombocytopenia. ${ }^{13}$ In contrast, other investigations reported that platelet function measured by hypotonic shock response, aggregation, or serotonin uptake is better maintained at $\mathrm{RT}^{14}$ and that cold storage leads to the loss of discoid shape and to the formation of large pseudopodia. ${ }^{15}$

Therefore, for the improvement of storage conditions and for the preparation of clinical studies, it is essential to shed more light upon the biochemical mechanisms associated with cold storage of platelets. Platelet reactivity to adenosine diphosphate (ADP) represents a significant characteristic of platelet integrity. The inhibition of ADP-induced aggregation is used as a major pharmacological principle for the treatment of cardiovascular diseases, for example, after stent implantation in coronary heart disease, associated with an increased risk of bleeding. ${ }^{16,17}$ ADP exerts its effects via the purinergic platelet receptors P2Y1, P2Y12, and P2X1. The receptors P2Y1 and P2Y12 are guanine nucleotidebinding protein (G-protein) coupled receptors, whereas $\mathrm{P} 2 \mathrm{X} 1$ is an adenosine triphosphate (ATP)-gated, nonselective cation channel. ${ }^{18} \mathrm{P} 2 \mathrm{Y} 1$ is a $\mathrm{G}_{\mathrm{q}}$-coupled receptor, activating platelet phospholipase $\mathrm{C}$, and stimulating calcium release from intracellular stores. ${ }^{19}$ P2Y12 inhibits platelet adenylyl cyclase through $\mathrm{G}_{\alpha \mathrm{i} .}{ }^{18}$ Simultaneous activation of both P2Y1 and P2Y12 results in platelet aggregation. ${ }^{20}$ Stimulation of the $\mathrm{P} 2 \mathrm{X} 1$ receptor alone causes a rapid calcium influx in platelets synergizing P2Y1 effects, but not inducing platelet aggregation. ${ }^{21}$

In this study, we analyzed the effects of cold storage on ADP-mediated responsiveness in apheresis-derived PC (APC) compared with room temperature storage, addressing purinergic receptor expression and function, aggregation responses, activation markers, and, in addition, inhibitory signaling pathways.

\section{Materials and Methods}

\section{Materials}

ADP was obtained from Haemochrom Diagnostica $\mathrm{GmbH}$ (Essen, Germany), thrombin receptor activating peptide-6 (TRAP-6) from BACHEM (Weil am Rhein, Germany). Mouse monoclonal fluorescein isothiocyanate (FITC)-conjugated antifibrinogen antibody was from STAGO Germany (Düssel- dorf, Germany) and mouse monoclonal FITC-conjugated antiCD62P antibody from Acris antibodies GmbH (Herford, Deutschland). Prostaglandin E1 (PGE1), diethylamine NONOate (DEA/NO), acetylsalicylic acid (ASS), probenecid, pluronic F-127, 4-[2-hydroxyethyl]-1-piperazineethanesulfonic acid (HEPES), apyrase, Ponceau S, and FITC-conjugated goat antirabbit antibody were from Sigma-Aldrich Chemie $\mathrm{GmbH}$ (Muenchen, Germany). Rabbit polyclonal anti-P2Y1, antiP2Y12, and anti-P2X1 antibodies were from Alomone Labs (Jerusalem, Israel). The selective P2Y1 receptor agonist [(1R,2R,3S,4R,5S)-4-[6-Amino-2-(methylthio)-9H-purin-9-

yl]-2,3-dihydroxy, bicycle [3.1.0] hex-1-yl]methyl] diphosphoric acid mono ester trisodium salt (MRS2365), the selective antagonist of P2Y1 $\left(1 R^{*}, 2 S^{*}\right)-4$-[2-Iodo-6-(methylamino)-9Hpurin-9-yl]-2-(phosphornooxy) bicyclo-[3.1.0]hexane-1methanol dihydrogen phosphate ester tetraammonium salt (MRS2500), the agonist of P2X1 receptor $\alpha, \beta$-methyleneadenosine 5 '-triphosphate trisodium salt ( $\alpha, \beta$-MeATP), and the potent P2X1 antagonist 4,4',4",4"'-[carbonylbis (imino5,1,3-benzenetriyl-bis (carbonylimino))] tetrakis-1,3-benzenedisul-fonic acid, octasodium salt (NF449) were from R\&D Systems GmbH (Wiesbaden-Nordenstadt Germany). Fluo4AM cell permeant was from Life Technologies $\mathrm{GmbH}$ (Darmstadt, Germany). Flow cytometric PLT vasodilatorstimulated phosphoprotein (VASP)/P2Y12 Kit for the measurement of P2Y12 receptor function was from STAGO GmbH (Duesseldorf, Germany). Mouse monoclonal phospho-VASP Ser $^{239}$ and phospho-VASP Ser ${ }^{157}$ antibodies were from Nanotools (Teningen, Germany). StarBright Blue 700 conjugated goat antirabbit and antimouse antibodies were from Bio-Rad Laboratories, Inc. (Muenchen, Germany).

\section{Blood and APC Collection}

Venous peripheral blood (PB) samples and apheresis-derived platelet concentrates (APCs) were obtained from informed healthy voluntary donors without any drug intake. Our studies with human platelets and the consent procedure were approved by our local ethics committee of the University of Wuerzburg (approval number: 101/15). The participants provided their written informed consent to participate in this study. The study was performed according to our institutional guidelines and to the Declaration of Helsinki.

PB was collected in polypropylene tubes containing 3.2\% citrate buffer (106 mM trisodium citrate, Sarstedt, Nuembrecht, Germany). APC pairs $\left(2.5 \times 10^{11}\right.$ platelets in $250 \mathrm{~mL}$ of plasma) were collected using Trima Accel devices with version 11.3 software and the Trima Accel LRS Platelet, Plasma Set (Terumo BCT, Lakewood, Colorado, United States). The ratio of inlet blood volume to anticoagulant (ACD-A) was 10:1. After preparation, APCs were stored either at room temperature (room temperature-stored APC, RT$\mathrm{APC}$ ) or at $4^{\circ} \mathrm{C}$ (cold-stored APC [C-APC]) for 6 days according to blood bank conditions on a standard agitator. On days 0 (23 hours after finalized apheresis), 2, and 5, samples from APC were taken for analysis under sterile conditions. For aggregation studies, samples were separately drawn on days 1 and 6 due to organizational reasons. Analysis of PB samples on day 0 was started within 1 hour after blood collection. 


\section{Blood Gas Analysis and Platelet Count}

Basic characteristics were detected with the blood gas system cobas b 123 POC, software version 4.14 (Roche Diagnostics $\mathrm{GmbH}$, Mannheim, Germany), platelet count with the hematology analyzer KX21N (Sysmex GmbH, Norderstedt, Germany).

\section{Preparation of Platelet-Rich Plasma and Washed Human Platelets}

Platelet-rich plasma (PRP) and washed platelets were prepared as described. ${ }^{22}$ Briefly, PRP was obtained by PB centrifugation at $280 \mathrm{~g}$ for 5 minutes. EGTA of $3 \mathrm{mM}$ was added to PRP or to samples from APC to prevent platelet activation. Subsequently, samples of PRP and APC were centrifuged at $430 \mathrm{~g}$ for 10 minutes. The pelleted platelets were washed once in CGS buffer (120 mM sodium chloride, $12.9 \mathrm{mM}$ trisodium citrate, $30 \mathrm{mM}$ D-glucose, $\mathrm{pH}$ 6.5) and resuspended in HEPES buffer $(150 \mathrm{mM} \mathrm{NaCl}, 5 \mathrm{mM} \mathrm{KCl}, 1 \mathrm{mM}$ $\mathrm{MgCl} 2,10 \mathrm{mM}$ D-glucose, and $10 \mathrm{mM}$ HEPES, pH 7.4) to a final concentration of $3 \times 10^{8}$ platelets $/ \mathrm{mL}$.

\section{Platelet Aggregation}

ADP-induced platelet aggregation of $10 \mu \mathrm{M}$ was measured in PRP or material from stored APC (diluted with plasma to platelet concentration of PRP) using an APACT 4004 aggregometer (LabiTec, Ahrensburg, Germany). Aggregation was measured for 5 minutes under continuous stirring at $1,000 \mathrm{rpm}$ and $37^{\circ} \mathrm{C}$. Maximal values were used for statistical calculations.

\section{Flow Cytometric Analysis}

Flow cytometric analysis was performed with PRP or with material from stored APC. For determination of basal and TRAP-6-stimulated fibrinogen binding, $15 \mu \mathrm{L}$ of PRP or APC samples, diluted with plasma to the platelet concentration in PRP, were stained with $15 \mu \mathrm{L}$ of FITC-conjugated antifibrinogen antibody or isotype control for 10 minutes at $37^{\circ} \mathrm{C}$. After that, the samples were stimulated for 2 minutes at $37^{\circ} \mathrm{C}$ with buffer (control) or $10 \mu \mathrm{M}$ TRAP-6.

For determination of basal and TRAP-6-stimulated CD62P expression, $30 \mu \mathrm{L}$ of PRP or APC samples, diluted with plasma to the platelet concentration in PRP, were stained with $3 \mu \mathrm{L}$ of FITC-conjugated anti-CD62P antibody or isotype control for 10 minutes at $37^{\circ} \mathrm{C}$. After that, the samples were stimulated for 2 minutes at $37^{\circ} \mathrm{C}$ with buffer (control) or $10 \mu \mathrm{M}$ TRAP-6.

The reactions were stopped for both fibrinogen- and CD62Pstained samples by fixation for 10 minutes at RT with $1 \%$ formaldehyde (final concentration). After that, they were diluted with $300 \mu \mathrm{L}$ of PBS/BSA/Glc and analyzed by flow cytometry.

For flow cytometric detection of purinergic receptor surface expression, $30 \mu \mathrm{L}$ of PRP or APC samples, diluted with plasma to the platelet concentration in PRP, were stained with $3 \mu \mathrm{L}$ of anti-P2Y1, anti-P2Y12, or anti-P2X1 antibodies or isotype control for 10 minutes at $37^{\circ} \mathrm{C}$. After that, the samples were stimulated for 2 minutes at $37^{\circ} \mathrm{C}$ with buffer (control) or $10 \mu \mathrm{M}$ TRAP-6. Samples were stopped with $1 \%$ formaldehyde (final concentration), fixed for 10 minutes at RT, and then centrifuged for 1 minute at
$14,000 \mathrm{~g}$. The pellet was resuspended in $100 \mu \mathrm{L}$ of PBS/ BSA/Glc (Dulbecco's PBS $\left(\mathrm{Ca}^{2+}, \mathrm{Mg}^{2+}\right.$ free), $5.5 \mathrm{mM}$ D-glucose, $0.5 \% \mathrm{BSA}$ ) and stained at RT in the dark for 30 minutes with $1 \mu \mathrm{L}$ of FITC-conjugated goat antimouse antibody. After that samples were diluted with $300 \mu \mathrm{L}$ of PBS/BSA/Glc and analyzed by flow cytometry.

Flow cytometric analysis was performed on a FACS Calibur flow cytometer from Becton Dickinson (Franklin Lakes, New Jersey, United States) using CELLQuest software, version 6.0.

The platelet population was identified by its forward and side scatter distribution and 10,000 events were analyzed for mean fluorescence.

\section{Platelet Preparation for the Measurement of P2Y1 Activity}

PGE1 of $500 \mathrm{nM}$ was added to PRP (as described for the preparation of washed platelets) or to material from stored APC and then centrifuged at $430 \mathrm{~g}$ for 10 minutes. The pellet was washed with $5 \mathrm{~mL}$ of modified Tyrode's buffer $(10 \mathrm{mM}$ HEPES, $150 \mathrm{mM} \mathrm{NaCl}, 3 \mathrm{mM} \mathrm{KCl}, 1 \mathrm{mM} \mathrm{MgCl}$, $5 \mathrm{mM}$ glucose and $0.1 \% \mathrm{BSA}, \mathrm{pH} 6.5$ ) containing $500 \mathrm{nM}$ PGE1. Platelets were resuspended in modified Tyrode's buffer without PGE1 and platelet concentration was adjusted to $0.6 \times 10^{8}$ platelets $/ \mathrm{mL}^{23}$

\section{Platelet Preparation for the Measurement of P2X1 Activity}

ASS of $1 \mathrm{mM}$ and $0.3 \mathrm{U} / \mathrm{mL}$ apyrase were added to PRP (as described for the preparation of washed platelets) or to material from stored APC and then centrifuged at $430 \mathrm{~g}$ for 10 minutes. The pellet was washed with $5 \mathrm{~mL}$ of modified Tyrode's buffer containing $1 \mathrm{mM}$ ASS and $0.3 \mathrm{U} / \mathrm{mL}$ apyrase. Platelets were resuspended in modified Tyrode's buffer containing $0.3 \mathrm{U} / \mathrm{mL}$ apyrase and platelet concentration was adjusted to $0.6 \times 10^{8}$ platelets $/ \mathrm{mL}^{23}$

\section{Measurement of P2Y1 and P2X1 Activity}

The activity of platelet purinergic P2Y1 and P2X1 receptors was measured by calcium flux-induced fluorescence in Fluo4AM-loaded platelets after selective stimulation. ${ }^{23}$ Briefly, in each well of a 96 -well black plate, $100 \mu \mathrm{L}$ of washed platelets were mixed with an equal volume of Hank's buffered saline solution (HBSS) containing $10 \mathrm{mM}$ HEPES, 0.1\% BSA, $2.5 \mathrm{mM}$ probenecid, $1 \mathrm{mM}$ EGTA, $0.01 \%$ pluronic acid, and $2 \mu \mathrm{M}$ Fluo4AM at $\mathrm{pH} 7.4$.

For the measurement of P2X1 activity, EGTA was substituted by $2.5 \mathrm{mM}$ calcium and apyrase was added to the final concentration of $0.3 \mathrm{U} / \mathrm{mL}$. The plate was incubated for 20 minutes at RT in the dark, followed by 20 minutes of incubation at $37^{\circ} \mathrm{C}$. During the last 10 minutes of incubation, $2 \mu \mathrm{L}$ of $100 \mu \mathrm{M}$ MRS2500, a P2Y1 antagonist, or $2 \mu \mathrm{L}$ of $100 \mu \mathrm{M}$ NF 449, a P2X1 antagonist, were added in negative controls. After measurement of the basal fluorescence (Ex 488, Em 538; 20 measurements at 1 second), platelets were stimulated with $2 \mu \mathrm{L}$ of $100 \mu \mathrm{M}$ MRS2365, a P2Y1 agonist, or $2 \mu \mathrm{L}$ of $100 \mu \mathrm{M} \alpha, \beta-$ MeATP, a P2X1 agonist. After stimulation, fluorescence signals were measured every second for the next 3 minutes using Fluoroscan Ascent Microplate Fluorometer from Fisher Scientific GmbH (Schwerte, Germany). 


\section{Measurement of P2Y12 Activity}

The activity of platelet P2Y12 receptor was measured by the flow cytometric PLT VASP/P2Y12 Kit. Briefly, aliquots of PB or APC, diluted with plasma to $3 \times 10^{8}$ platelets $/ \mathrm{mL}$ were stimulated with PGE1 alone or with a combination of PGE1 and ADP at RT. After stimulation, samples were fixed and stained as described in the manufacturer's instructions, followed by flow cytometric measurement of fluorescence. Platelet reactivity index (PRI) was calculated using corrected mean fluorescence intensities (MFIc) as PRI $=[$ MFIc (PGE1) MFIc (PGE1 + ADP)] / [MFIc (PGE1)] × 100\%.

\section{Western Blot Analysis}

VASP phosphorylation in washed platelets was determined by Western blot analysis. For this purpose, $100 \mu \mathrm{L}$ of washed platelet suspension was supplemented with $1 \mathrm{mM} \mathrm{CaCl}$, followed by stimulation with buffer, $5 \mathrm{nM}$ DEA/NO, or $5 \mathrm{nM}$ PGE1 for 2 minutes at $37^{\circ} \mathrm{C}$. The cell lysates were loaded onto the gel, separated by sodium dodecyl sulfate polyacrylamide gel electrophoresis (SDS-PAGE) and then transferred onto nitrocellulose membranes. The membranes were incubated with mouse monoclonal phospho-VASP $\operatorname{Ser}^{239}$ (Clone 16C2) and phospho-VASP Ser ${ }^{157}$ (Clone 5C6) antibodies overnight at $4^{\circ} \mathrm{C}$. For visualization of the signal, goat antimouse immunoglobulin (Ig)-G conjugated with StarBright Blue 700 was used as secondary antibody, followed by detection with Chemidoc MP imaging system (Bio-Rad Laboratories, Inc., Hercules, California, United States) and analysis with the corresponding Image Laboratory Software Version 6.0.

\section{CAMP and CGMP Measurements}

Cyclic adenosine monophosphate (cAMP) and cyclic guanosine monophosphate (cGMP) in washed platelets were detected by cAMP (enzyme-linked immunoassay) ELISA Kit and GMP ELISA Kit, respectively, following the manufacturer's instructions (Cayman Chemical, Hamburg, Germany).

\section{Statistical Analysis}

Descriptive data were calculated with the MedCalc statistic program (MedCalc Software bvba, Mariakerke, Belgium) and GraphPad PRISM 7 (GraphPad Software, San Diego, California, United States). Data distribution analysis was performed with Shapiro-Wilk test. Differences of variances between groups were analyzed by one-way analysis of variance (ANOVA) followed by post hoc Tukey-Kramer test. A $p<0.05$ was considered statistically significant, $p<0.1$ as tendency.

\section{Results}

\section{ADP-Induced Aggregation Preservation in Cold-Stored Platelets}

In freshly prepared APCs, $10 \mu \mathrm{M}$ ADP-induced light transmission aggregation showed values of approximately $80 \%$, comparable to PRP of PB samples (-Fig. 1). The aggregation responses in RT-APC were dramatically decreased on day 1 and completely declined until day 6 . In contrast, aggregation

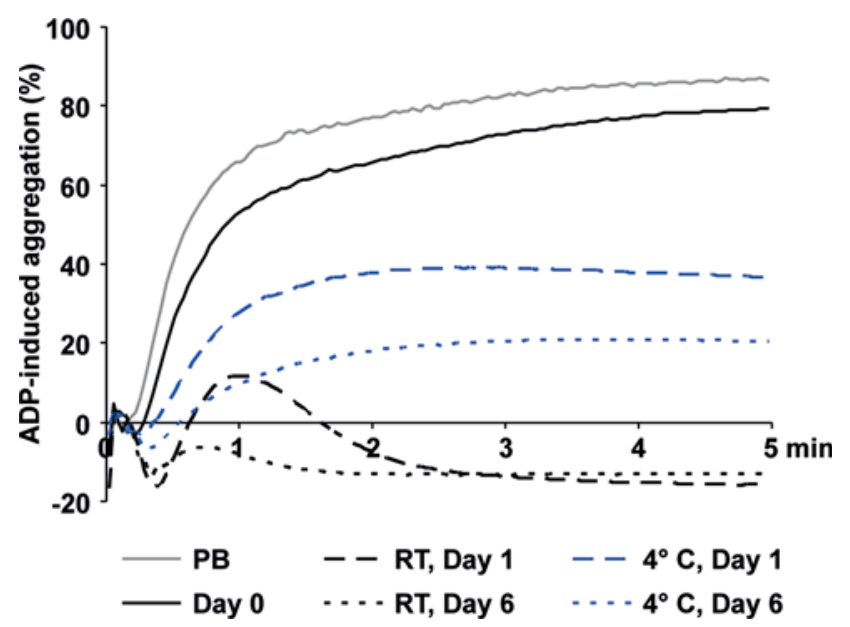

Fig. 1 ADP-induced aggregation under RT and cold storage. PRP of fresh PB samples, of RT-APC and of C-APC was used to measure light transmission aggregation after stimulation with $10 \mu \mathrm{M}$ ADP. Mean aggregation curves are shown. $n=6$. ADP, adenosine diphosphate; $C$ APC, cold-stored apheresis-derived platelet concentrates; RT, room temperature.

was partially maintained in C-APC with $36.6 \pm 11.9 \%$ on day 1 and $20.4 \pm 12.1 \%$ on day 6 .

\section{Preactivation Marker Increase under Cold Storage}

The reactivity and the preactivation status of stored platelets were analyzed by basal and induced fibrinogen binding and CD62 expression (-Fig. 2). Upon stimulation with $10 \mu \mathrm{M}$ TRAP-6, fibrinogen binding in PB samples and in APCs increased from $21.7 \pm 1.0$ to $408.7 \pm 100.9 \mathrm{MFI}$, and from $23.0 \pm 1.5$ to $359.1 \pm 67.7 \mathrm{MFI}$, respectively ( - Fig. $2 \mathrm{~A}$ ). On days 2 and 5 , the basal values remained unchanged in RTAPC, but increased to $65.4 \pm 17.3$ and $86.8 \pm 31.0 \mathrm{MFI}$ in CAPC. TRAP-6-stimulated values decreased in the course of storage to $216.5 \pm 58.9 \mathrm{MFI}$ on day 2 and to $203.4 \pm 54.1 \mathrm{MFI}$ on day 5 for RT-APC, and comparably to $254.7 \pm 67.3 \mathrm{MFI}$ and $238.4 \pm 69.0 \mathrm{MFI}$ in C-APC. Basal CD62P expression rose during storage of C-APC from $20.6 \pm 0.8$ MFI to $93.1 \pm 8.9$ MFI until day 5 , and to $44.9 \pm 5.9$ MFI in RT-APC on day 5 (-Fig. 2B). The increment of TRAP-6-stimulated CD62P expression was decreasing until day 5 under both storage conditions, with $454.5 .7 \pm 49.0$ MFI on day 0 and $283.6 \pm 22.8$ MFI on day 5 for RT-APC, and $260.6 \pm 30.7$ MFI on day 5 for C-APC. Representative flow cytometry dot plot diagrams of sideward light scatter (SSC) versus FITC fluorescence (FL1) illustrate the shift of unstimulated platelets toward elevated fibrinogen binding and CD62P expression in C-APC in contrast to RT-APC ( - Fig. 2C and D).

\section{Basic Characteristics of C-APC and RT-APC}

C-APC developed a slight reduction of platelet concentration and a mild increase of potassium levels during storage in comparison to RT-APC ( - Table $\mathbf{1}$ ). The pH levels measured at $22^{\circ} \mathrm{C}$ showed a rising tendency, more pronounced in RT-APC. Glucose consumption and lactate generation was stronger in RT-APC, whereas annexin $\mathrm{V}$ binding remained unchanged during storage at both temperatures. 
A

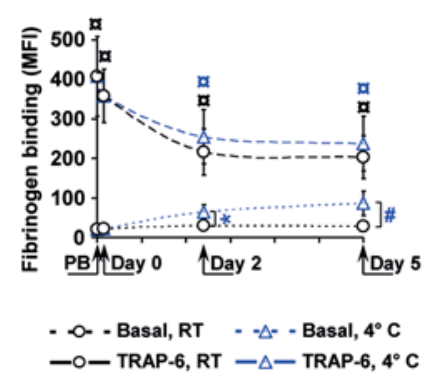

B

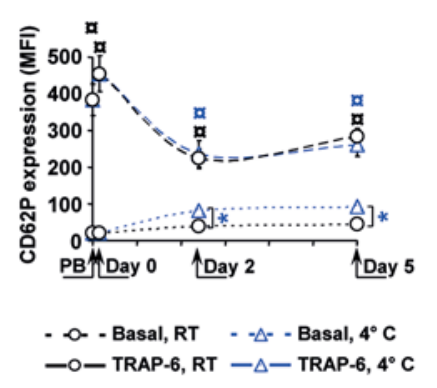

C

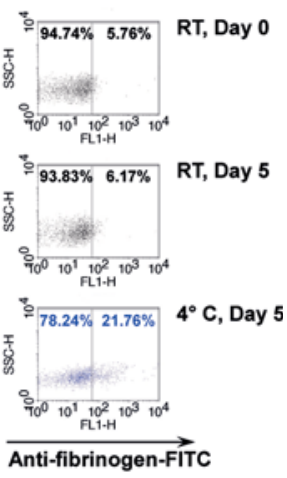

D

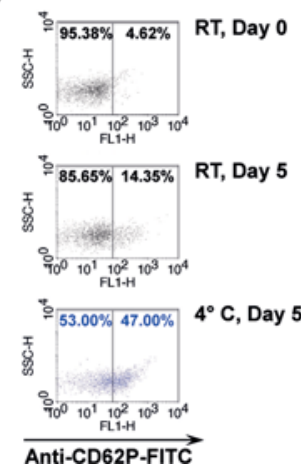

Fig. 2 CD62P expression and fibrinogen binding. Basal or $10 \mu \mathrm{M}$ TRAP-6induced CD62P expression (A) and fibrinogen binding (B) were measured by flow cytometry at different time points as indicated. Representative scatter diagrams illustrate the distribution of platelets detected by FITC-conjugated antifibrinogen antibodies (C) or anti-CD62P antibodies (D). $n=4$; mean \pm SEM; ${ }^{*} p<0.1$ or ${ }^{*} p<0.05$, compared with RT-APC at the same time points; ${ }^{a} p<0.05$, compared with basal values (for RT-APC in black, for C-APC in blue). C-APC, cold-stored apheresis-derived platelet concentrates; $\mathrm{MFI}$, mean fluorescence intensities; RT-APC, room temperature APC; SEM, standard error of mean; TRAP-6, thrombin receptor activating peptide.

\section{Purinergic Receptor Expression Increases, but the Function of P2Y1 and P2X1 Decreases during Cold Storage}

Since ADP responsiveness of platelets is mediated via purinergic receptors, the expression and function of P2Y1, P2X1 and P2Y12 receptors were analyzed ( - Fig. 3 ). In fresh APC, the basal expression remained unchanged for all three receptor types compared with PB ( $\mathbf{F i g . ~} 3 \mathbf{A}-\mathbf{C}$ ). In contrast to RT-APC with stable values throughout storage, the basal expression slightly increased in C-APC until day 5, by $31 \%$ for P2Y1, by $37 \%$ for P2X1, and by $43 \%$ for P2Y12. Initially, TRAP-6 stimulated purinergic receptor expression by approximately four folds. In the course of storage, induced P2Y1 expression decreased by $20 \%$ in both APC types. P2X1 expression was comparably maintained, whereas P2Y12 expression dropped in RT-APC by $25 \%$ until day 5 in contrast to stable levels in C-APC.

P2Y1 function, measured by calcium-induced fluorescence after selective stimulation, showed a progressive decline in C-APC from $0.69 \pm 0.10$ to $0.26 \pm 0.02$ relative fluorescence units (RFU) on day 2 and to $0.20 \pm 0.01 \mathrm{RFU}$ on day 5. In RT-APC, this effect was less emphasized with $0.42 \pm 0.05 \mathrm{RFU}$ on day 2 and $0.31 \pm 0.02 \mathrm{RFU}$ on day 5 (-Fig. 3D). P2X1-related calcium-induced fluorescence remained almost unaffected during storage in RT-APC, but dropped in C-APC from $0.65 \pm 0.08$ to $0.22 \pm 0.01 \mathrm{RFU}$ on day 2 and $0.22 \pm 0.02 \mathrm{RFU}$ on day 5 (- Fig. 3E). The PRI levels indicating P2Y12 activity were stable with approximately $80 \%$ throughout storage in RT-APC, comparable to PB samples. However, PRI levels in C-APC dropped to $60.1 \pm 7.7 \%$ on day 2 and to $56.4 \pm 4.6 \%$ on day 5 ( $\mathbf{- F i g . ~} \mathbf{3 F}$ ).

\section{P2Y12 Function is Maintained in Cold-Stored Platelets}

The PRI is a calculated parameter using PGE1-induced VASP phosphorylation levels without or with additional ADP stimulation (- Fig. 4). In contrast to RT-APC, PGE1-induced VASP phosphorylation was significantly decreased in C-APC reaching $57.0 \pm 9.3$ corrected mean fluorescence intensity (MFIc) on day 2 and $62.7 \pm 14.4$ MFIc on day 5 of storage (-Fig. 4A). The additional stimulation with ADP led to an excessive decrease of VASP phosphorylation to levels as measured in RT-APC (-Fig. 4B), indicating an unaffected functional activity of the P2Y12 receptor.

\section{VASP Phosphorylation is Less Inducible in Cold-Stored Platelets}

The inhibitory pathways of platelets were investigated by Western blot analysis of VASP phosphorylation at $\operatorname{Ser}^{239}$ and

Table 1 Basic characteristics of C-APC and RT-APC

\begin{tabular}{|l|l|l|l|l|l|l|}
\hline Parameter & Unit & APC (day 0) & RT-APC (day 2) & RT-APC (day 5) & C-APC (day 2) & C-APC (day 5) \\
\hline Platelets & $\times 10^{3} / \mathrm{\mu L}$ & $1,148 \pm 49$ & $1,145 \pm 68$ & $1,132 \pm 66$ & $1,006 \pm 126^{\mathrm{a}, \mathrm{c}}$ & $952 \pm 145^{\mathrm{a}, \mathrm{c}}$ \\
\hline Potassium & $\mathrm{mmol} / \mathrm{L}$ & $3.18 \pm 0.08$ & $3.24 \pm 0.04$ & $3.26 \pm 0.04^{\mathrm{a}}$ & $3.45 \pm 0.06^{\mathrm{b}, \mathrm{d}}$ & $3.52 \pm 0.03^{\mathrm{b}, \mathrm{d}}$ \\
\hline Annexin $\mathrm{V}$ & $\mathrm{MFI}$ & $17.3 \pm 2.4$ & $18.2 \pm 1.5$ & $21.5 \pm 1.4$ & $18.2 \pm 2.4$ & $18.1 \pm 1.0$ \\
\hline $\mathrm{pH}\left(22^{\circ} \mathrm{C}\right)$ & - & $7.37 \pm 0.03$ & $7.60 \pm 0.02^{\mathrm{b}}$ & $7.46 \pm 0.03$ & $7.47 \pm 0.02^{\mathrm{a}, \mathrm{d}}$ & $7.45 \pm 0.02^{\mathrm{a}}$ \\
\hline Glucose & $\mathrm{mg} / \mathrm{dL}$ & $399 \pm 36$ & $369 \pm 39^{\mathrm{b}}$ & $329 \pm 44^{\mathrm{b}}$ & $388 \pm 37^{\mathrm{b}, \mathrm{d}}$ & $372 \pm 41^{\mathrm{b}, \mathrm{d}}$ \\
\hline Lactate & $\mathrm{mmol} / \mathrm{L}$ & $1.90 \pm 0.37$ & $4.63 \pm 0.32^{\mathrm{b}}$ & $9.03 \pm 0.47^{\mathrm{b}}$ & $3.43 \pm 0.29^{\mathrm{b}, \mathrm{d}}$ & $5,90 \pm 0.21^{\mathrm{b}, \mathrm{d}}$ \\
\hline
\end{tabular}

Abbreviations: C-APC, cold-stored apheresis-derived platelet concentrates; MFI: mean fluorescence intensity (in arbitrary units);RT-APC, room temperature APC.

${ }^{\mathrm{a}} p<0.1$, compared with day 0 .

${ }^{\mathrm{b}} p<0.05$, compared with day 0 .

${ }^{c} p<0.1$, compared with the corresponding day of storage.

${ }^{d} p<0.05$, compared with the corresponding day of storage; mean \pm standard error of mean; $n=3$. 
A

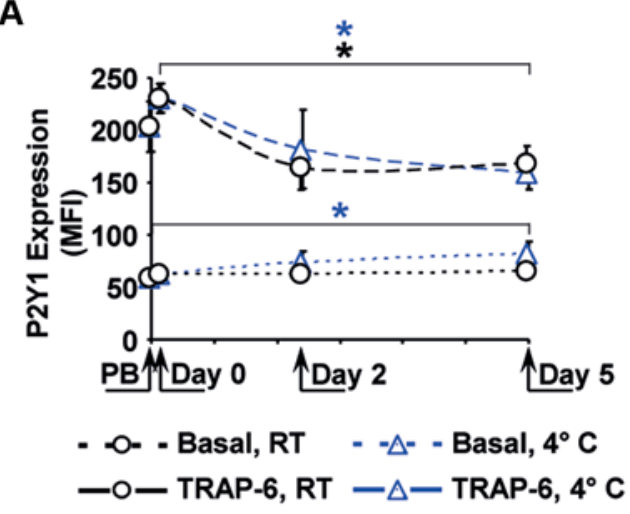

B

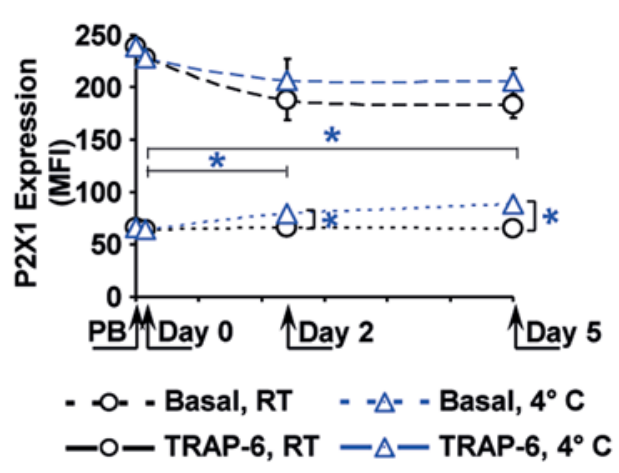

C

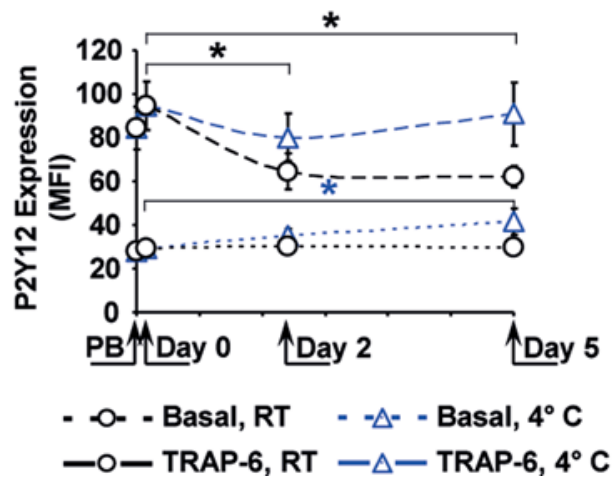

D

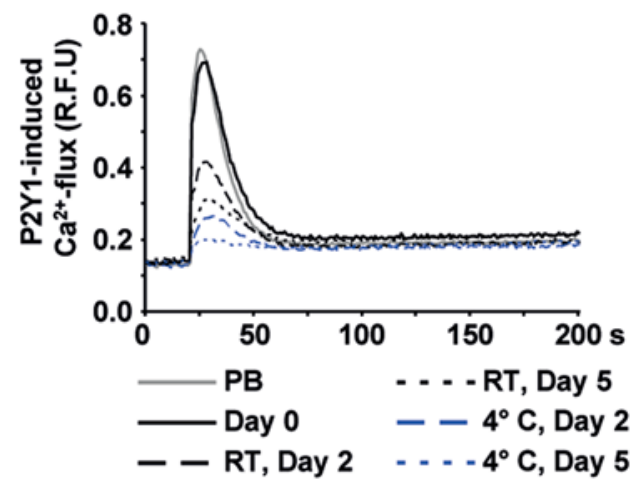

E

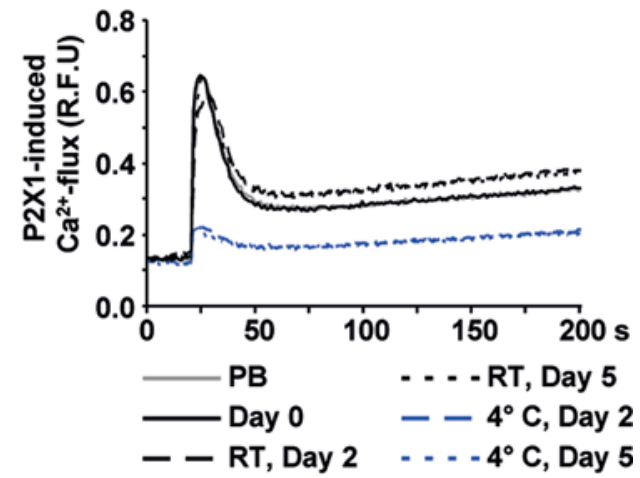

$\mathbf{F}$

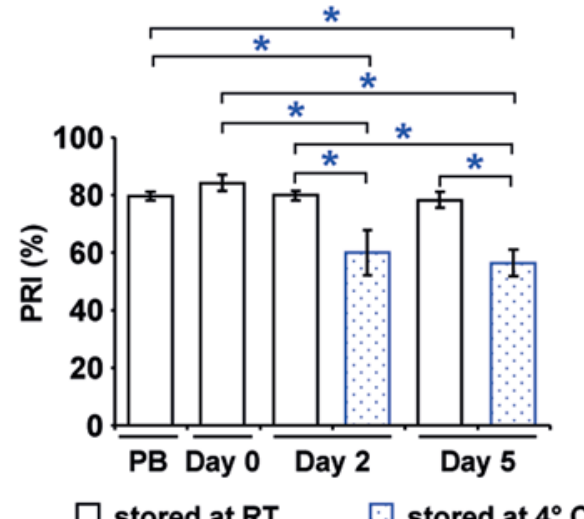

Fig. 3 Expression and function of purinergic receptors. The mean fluorescence intensities (MFI) of basal or $10 \mu \mathrm{M}$ TRAP-6-stimulated receptor expression, detected by flow cytometry, are shown for P2Y1 (A), P2Y12 (B) and P2X1 (C). The function of the P2Y1 (D) and of the P2X1 (E) receptor was measured by calcium-induced fluorescence after selective stimulation, shown as mean values in relative fluorescence units (RFU). The function of the P2Y12 receptor (F) is expressed as platelet reactivity index (PRI) determined by the flow cytometric VASP assay. Mean \pm SEM; $n=6$; ${ }^{p} p<0.05$ (for RT-APC in black, for C-APC in blue). C-APC, cold-stored apheresis-derived platelet concentrates; PB, peripheral blood; RT, room temperature; SEM, standard error of mean; TRAP-6, thrombin receptor activating peptide; VASP, vasodilator-stimulated phosphoprotein.

Ser $^{157}$ using the nitric oxide (NO) donor diethylamine NONOate (DEA/NO) and prostaglandin E1 (PGE1; - Fig. 5). In RTAPC, 5 nM DEA/NO and PGE1 similarly provoked a 4-5-fold increase of phosphorylation at $\mathrm{Ser}^{239}$ with increasing tendency during storage ( - Fig. 5A). In C-APC, the increments at $\mathrm{Ser}^{239}$ were smaller, with a 1.8 -fold elevation on day 2 and a 1.6-fold elevation on day 5 for DEA/NO stimulation, and 1.5fold and 1.6-fold PGE1 stimulation, respectively. At Ser ${ }^{157}$, phosphorylation levels were similarly stimulated in RT-APC, up to three folds under DEA/NO or four folds under PGE1 compared with basal values (-Fig. 5B). In C-APC, induced phosphorylation was less emphasized, with no significant increase on both investigated days for DEA/NO stimulation and a significant, but only two-fold shift on day 2 for PGE1.

\section{Stimulation of Cyclic Nucleotide Levels is Dampened under Cold Storage}

The cyclic nucleotide levels (cAMP and cGMP) were additionally measured to complement data from Western blot analysis (-Fig. 6). In RT-APC, DEA/NO increased cGMP concentrations more than four folds on day 0 , comparable to $\mathrm{PB}$ samples, 8.5 folds on day 2 and 11 folds on day 5 ( - Fig. 6A). In 
A

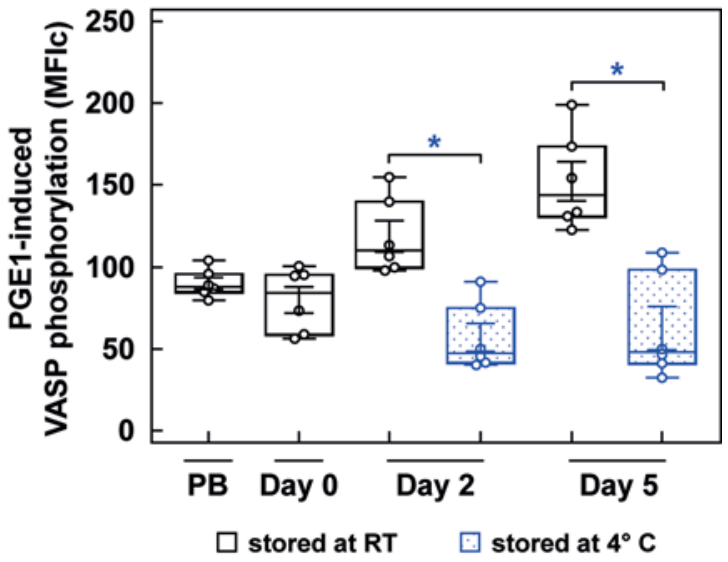

B

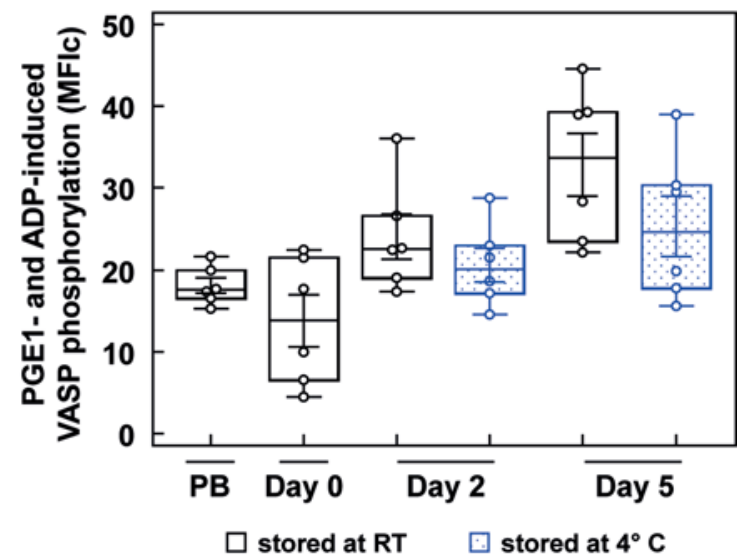

Fig. 4 PRI-related VASP phosphorylation measured by flow cytometry. The box-and-whisker plots show the distribution of corrected mean fluorescence intensities after stimulation with PGE1 (A) and after stimulation with PGE1 + ADP (B) of PB samples, RT-APC and CAPC (blue color) as indicated; Analysis was performed by flow cytometry. Results are presented as mean \pm SEM; $n=6 ;{ }^{*} p<0.05$. ADP, adenosine diphosphate; C-APC, cold-stored apheresis-derived platelet concentrates; MFI, mean fluorescence intensities; PB, peripheral blood; PGE1, prostaglandin E1; PRI, platelet reactivity index; RT, room temperature; SEM, standard error of mean; VASP, vasodilator-stimulated phosphoprotein.

contrast, DEA/NO was not able to relevantly enhance cGMP levels in C-APC. PGE1 did not affect cGMP values, but led to an elevation of CAMP levels up to two folds on days 2 and 5 in RTAPC (-Fig. 6B). The increment of cAMP concentrations continuously decreased during storage of C-APC. For the stimulation with DEA/NO, a weak induction of cAMP levels by $50 \%$ was observed in RT-APC at all time points of storage, but not in C-APC.

\section{The Inhibitory Effect of DEA/NO and PGE1 is Reduced in Cold-Stored Platelets}

DEA/NO- or PGE1-mediated inhibition of ADP-induced aggregation is attenuated in platelets from PB samples stored as citrated whole blood at $4^{\circ} \mathrm{C}$ for 24 hours ( - Fig. 7). Compared with fresh $\mathrm{PB}$ samples with unaffected aggregation under $5 \mathrm{nM}$ DEA/NO (2.9\% inhibition) and almost complete inhibi-
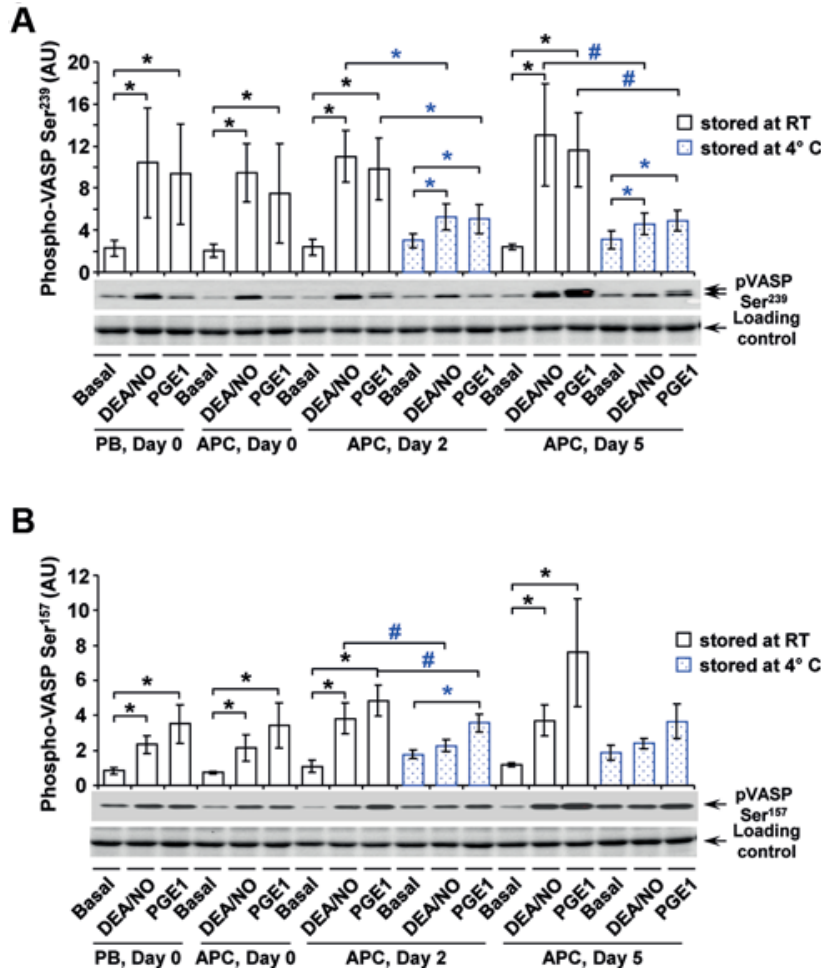

Fig. 5 Western blot analysis of basal and induced VASP phosphorylation. Quiescent washed platelets from fresh PB samples and from stored RT-APC or C-APC, without or after stimulation with DEA/NO or PGE1 as indicated, were lysed with Laemmli's buffer and analyzed on Western blot for VASP Ser ${ }^{239}$ (A) and VASP Ser ${ }^{157}$ (B) phosphorylation. After scanning bands were quantified by the Image Lab program. Results are presented in arbitrary units (AU) as mean \pm SEM; $n=5$; ${ }^{*} p<0.05,{ }^{\#} p<0.1$ (compared as indicated; blue: RT-APC to C- APC). C-APC, cold-stored apheresis-derived platelet concentrates; DEA/NO, diethylamine NONOate; $\mathrm{PB}$, peripheral blood; $\mathrm{PGE1}$, prostaglandin $\mathrm{E} 1$; RT, room temperature; SEM, standard error of mean; VASP, vasodilator-stimulated phosphoprotein.

tion (more than $88.5 \%$ inhibition) under $1,000 \mathrm{nM}$, DEA/NO resulted in $20.5 \pm 11.6 \%$ inhibition at $5 \mathrm{nM}$ and $70.0 \pm 14.6 \%$ inhibition at $1,000 \mathrm{nM}$ after 24 hours of RT-storage (-Fig. 7A). Under cold storage for 24 hours, the inhibition of aggregation was dramatically reduced to $3.6 \pm 3.0 \%$ at $5 \mathrm{nM}$ DEA/NO and $13.3 \pm 5.4 \%$ at $1,000 \mathrm{nM}$ DEA/NO compared with the extent of inhibition in freshly drawn, 1 hour cold or 24 hours RT-stored samples. The onset of tampered inhibition is observable after 1 hour of cold storage with significantly weaker inhibition of aggregation by $76.3 \pm 5.7 \%$ at $1,000 \mathrm{nM} \mathrm{DEA} / \mathrm{NO}$ in comparison to inhibition of ADPinduced aggregation in freshly prepared $\mathrm{PB}$ samples.

PGE1 of $125 \mathrm{nM}$ led to $64.0 \pm 14.6 \%$ inhibition of ADPinduced aggregation, whereas 250 and $500 \mathrm{nM}$ PGE1 almost completely blocked it in fresh PB ( - Fig. 7B). In samples stored for 24 hours at RT, all investigated PGE1 concentrations prevented ADP-induced platelet aggregation, whereas cold storage was associated with lower inhibitory effects after 1 hour with $65.6 \pm 12.6 \%$ at $125 \mathrm{nM}$ and with $87.6 \pm 3.1 \%$ at $250 \mathrm{nM}$ PGE1, and after 24 hours even more pronounced with $52.3 \pm 7.4 \%$ or $73.7 \pm 4.6 \%$, respectively. At the high 500 nM PGE1 concentration, there was no reduction 
A

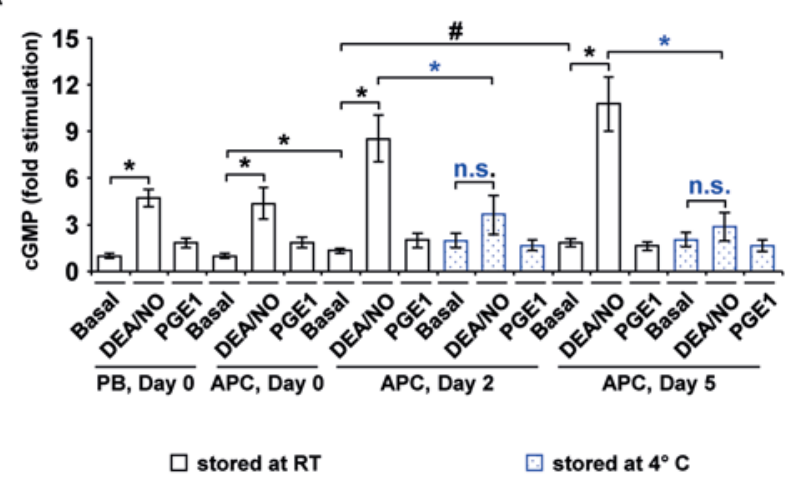

B

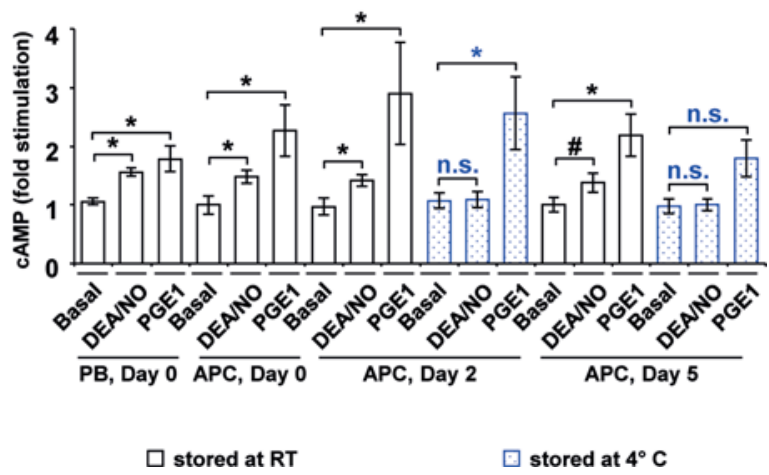

Fig. 6 Cyclic nucleotide levels. Cyclic nucleotide concentrations were measured in lysed washed platelets from fresh PB samples and from stored RT-APC or C-APC. After extraction with diethyl ether the contents of CGMP (A) and CAMP (B) were determined with immunoassays. Results are presented as mean (fold stimulation) $\pm \mathrm{SEM} ; n=4$; ${ }^{*} p<0.05,{ }^{*} p<0.1$ (compared as indicated; blue: RT-APC to C-APC; $n$. s.: not significant). APC, apheresis-derived platelet concentrates; CAMP, cyclic adenosine monophosphate; C-APC, cold-stored apheresis-derived platelet concentrates; cGMP, cyclic guanosine monophosphate; DEA/NO, diethylamine NONOate; PB, peripheral blood; PGE1, prostaglandin E1; RT, room temperature; SEM, standard error of mean.

of inhibition after 1 hour and it was only slightly tampered with $85.1 \pm 2.7 \%$ after 24 hours at cold temperature compared with fresh PB samples with $89.5 \pm 3.5 \%$.

\section{Discussion}

The minimization of storage lesions by optimization of storage conditions is an important issue of manufacturing platelet concentrates. ${ }^{24-26}$ In this context, it was the intention to analyze the effects of cold temperature on ADPdependent responsiveness, a system playing an important role for physiological platelet integrity and used as pharmacological target to suppress thrombus formation. ${ }^{16,17}$

The study confirms that ADP-induced platelet aggregation is better maintained at $4^{\circ} \mathrm{C}$ in comparison to RT..$^{25,27,28}$ The molecular and biochemical investigations have revealed that the mechanisms for increased ADP responses are related to cold-induced attenuation of inhibitory signaling rather than to differences in expression or function of purinergic receptors. In general, cold storage leads to preactivation of plate-
A

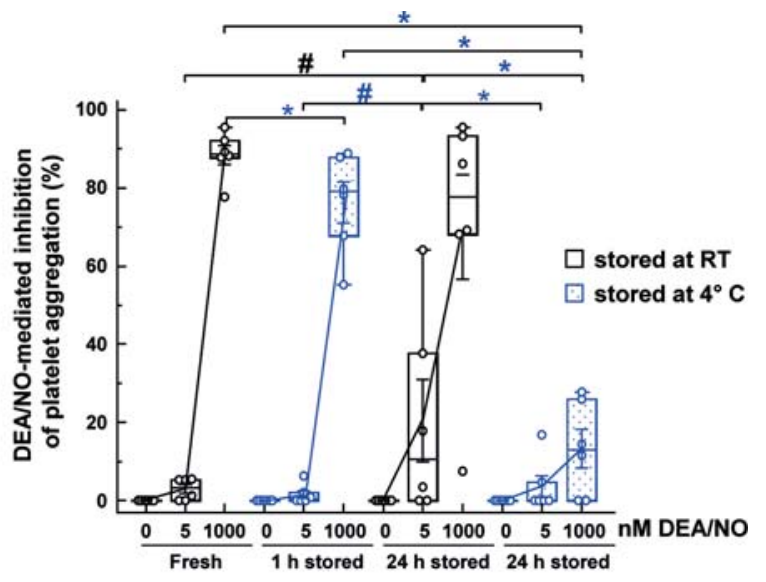

B

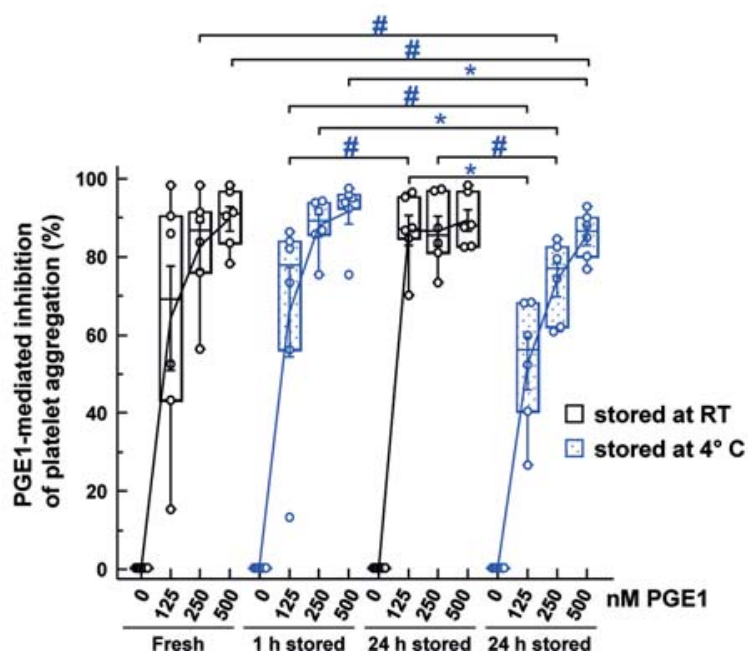

Fig. 7 Inhibitory effects of DEA/NO and PGE1 on ADP-induced aggregation in RT or cold-stored PB samples. Light transmission aggregation was performed with PRP of stored PB samples as indicated. The results are shown as mean $\% \pm$ SEM and represent the relative inhibition of $10 \mu \mathrm{M}$ ADP-induced aggregation in relation to the corresponding PB samples without DEA/NO or PGE1 incubation (as $0 \%$ inhibition); $n=6 ;{ }^{*} p<0.05,{ }^{*} p<0.1$ (compared as indicated; blue: RTAPC to C-APC). ADP, adenosine diphosphate; C-APC, cold-stored apheresis-derived platelet concentrates; DEA/NO, diethylamine NONOate; PB, peripheral blood; PGE1, prostaglandin E1; RT, room temperature; SEM, standard error of mean.

lets ${ }^{9,11}$ as illustrated by elevated basal levels of fibrinogen binding and CD62P expression. Hoffmeister et al could show that rearrangements of the GPIb surface configuration are initiated by cold temperature, leading to its clustering. ${ }^{29}$ Therefore, it would be of interest, if purinergic receptors experience a similar variation of their assembly on the platelet surface. An essential influence of refrigeration on purinergic receptor expression, however, was not observable, although the surface content of these receptors increase upon agonist-induced platelet activation. ${ }^{30}$

Surprisingly, the functional activity of P2Y1 and P2X1 measured as calcium-induced fluorescence was even less maintained at cold storage. Earlier studies have also shown that refrigeration disturbs the internal calcium regulation of platelets. ${ }^{31}$ Instead, the P2Y12 receptor appears to be more robust to storage lesion at $\mathrm{RT}^{32}$ and similarly at $4^{\circ} \mathrm{C}$. The 
coupling of the receptor to intraplatelet signaling pathways other than P2Y1 and P2X1, without association to calcium release, may play a role for that phenomenon. In the context of PRI determination, it is required to closely study the underlying shifts of VASP phosphorylation. ${ }^{33,34}$ Thereby, it was evident that stimulation of PGE1-induced VASP phosphorylation in C-APC is decreased, whereas ADP-induced inhibition of VASP phosphorylation remains intact, indicating maintained P2Y12 receptor function.

PGE1 stimulates one of the two main inhibitory pathways in platelets via the platelet IP (prostacyclin) receptor, coupled to $\mathrm{G}_{\mathrm{s}}$-activating protein of membrane-associated adenylyl cyclase (AC). AC activation leads to the increase of intracellular CAMP concentration followed by enhanced cAMP-mediated VASP phosphorylation. ${ }^{3,36}$

The second inhibitory pathway in platelets is activated by NO produced by various NO synthase-containing cells or released by chemical compounds like DEA/NO. ${ }^{35,36}$ NO permeates through the platelet membrane and directly activates soluble guanylyl cyclase (sGC) in the cytosol. This results in the stimulation of cGMP production and, consecutively, in enhanced cGMP-mediated VASP phosphorylation. ${ }^{35,36}$ In C-APC, VASP phosphorylation was less inducible at both $\mathrm{Ser}^{239}$ and at $\mathrm{Ser}^{157}$ after PGE1 and DEA/NO stimulation. Accordingly, intracellular levels of cAMP and cGMP, stimulated with PGE1 or DEA/NO, were reduced in C-APC compared with RT-APC.

Despite different molecular mechanisms and different substrates like ATP and GTP, both inhibitory pathways in platelets are affected by cold storage. However, the key enzymes AC and sGC possess the same pyrophosphatelyase (cyclizing) activity, (ATP pyrophosphatelyase [cyclizing], EC 4.6.1.1) and (GTP pyrophosphatelyase [cyclizing], EC 4.6.1.2), ${ }^{37}$ consistent with cognate thermosensitive characteristics. In former studies, it was demonstrated that the increase of temperature for enzyme incubation from low to higher than $25^{\circ} \mathrm{C}$ led to an abrupt decrease of energy of AC activation. ${ }^{38}$ The enzyme stability and the rate of AC activation from Neurospora grassa and turkey erythrocytes were also temperature and membrane lipid composition (fluidity) dependent. ${ }^{39,40}$ Similar to AC, GC enzyme activities from various species (human embryonic kidney cells [HEK-293T], Caenorhabditis elegans, and monkey fibroblast-like cell line Cos-7) were sensitive to temperature variations with activity peaks between 15 and $30^{\circ} \mathrm{C} .^{41-43}$ Platelet storage at $4^{\circ} \mathrm{C}$ for more than 24 hours may have an impact not only on enzyme kinetic, but also induce a long-term change of membrane and surrounding proteins fluidity. These effects may result in irreversible dysfunction of pyrophosphatelyase (cyclizing) activity of AC and GC, reduced CAMP and cGMP synthesis, and as a consequence, in impaired VASP phosphorylation and platelet inhibition.

\section{Limitations}

As a limitation, it should be mentioned that the results refer to an in vitro study with platelets separated by apheresis and stored in autologous plasma. However, platelet concentrates can be manufactured with variable methods, for example, as pooled concentrates, with the use of additive solutions or treated by pathogen inactivation procedures. ${ }^{44,45}$ The impact of cold storage on ADP-dependent platelet function in such product modifications should be subject of additional studies. In this study, the standard ACD-A solution was used as the common anticoagulant in transfusion medicine, albeit the citrate-induced reduction of ionized calcium potentially interferes with platelet function. Thrombin inhibitors-like hirudin may be alternatives, but there is evidence that thrombin inhibitors also affect inhibitory pathways, thereby enhancing VASP phosphorylation and dampening platelet reactivity. ${ }^{34}$ Interestingly, BAPA (benzylsulfonyl-D-Arg-Pro4-amidinobenzylamide) represents a dual inhibitor of factor $\mathrm{Xa}$ and thrombin, was able to maintain platelet aggregation and function in stored blood samples at RT better than citrate, indicating a significant role of the anticoagulant in platelet preservation. ${ }^{46,47}$

\section{Conclusion}

In conclusion, cold-stored platelets possess a higher sensitivity to ADP despite impairment of P2Y1 and partially of P2X1 function. Attenuated inhibitory signaling with early onset during cooling is a major factor promoting activation and ADP-induced aggregation of platelets compared with RTAPC. Future studies should address time-dependent effects of refrigeration on ADP-mediated platelet integrity and the reversibility of cold induced storage lesions to further improve manufacturing processes of platelet concentrates and to facilitate the design of clinical trials with cold-stored platelet products.

\section{Funding}

This publication was supported by the Open Access Publication Fund of the University of Wuerzburg. The study was funded by the Deutsche Forschungsgemeinschaft (DFG, German Research Foundation), project number: KO 5256/3-1 (J.K.) and KO 5294/2-1 (A.Kob.).

Conflict of Interest

None declared.

\section{References}

1 Kaufman RM, Djulbegovic B, Gernsheimer T, et al; AABB. Platelet transfusion: a clinical practice guideline from the AABB. Ann Intern Med 2015;162(03):205-213

2 Cauwenberghs S, van Pampus E, Curvers J, Akkerman JW, Heemskerk JW. Hemostatic and signaling functions of transfused platelets. Transfus Med Rev 2007;21(04):287-294

3 Hegde S, Akbar H, Zheng Y, Cancelas JA. Towards increasing shelf life and haemostatic potency of stored platelet concentrates. Curr Opin Hematol 2018;25(06):500-508

4 Stubbs JR, Tran SA, Emery RL, et al. Cold platelets for traumaassociated bleeding: regulatory approval, accreditation approval, and practice implementation-just the "tip of the iceberg". Transfusion 2017;57(12):2836-2844

5 Murphy S, Gardner FH. Effect of storage temperature on maintenance of platelet viability-deleterious effect of refrigerated storage. N Engl J Med 1969;280(20):1094-1098

6 Slichter SJ. Controversies in platelet transfusion therapy. Annu Rev Med 1980;31:509-540 
7 Strandenes GK, Bjerkvig EK, Fosse CK, et al. Cold-stored apheresis platelets in treatment of postoperative bleeding in cardiothoracic surgery. Transfusion 2016;56(S4):S29-020B

8 Krachey E, Viele K, Spinella PC, Steiner ME, Zantek ND, Lewis RJ. The design of an adaptive clinical trial to evaluate the efficacy of platelets stored at low temperature in surgical patients. J Trauma Acute Care Surg 2018;84(6S, suppl 1):S41-S46

9 Sandgren P, Shanwell A, Gulliksson H. Storage of buffy coatderived platelets in additive solutions: in vitro effects of storage at 4 degrees C. Transfusion 2006;46(05):828-834

10 Sandgren P, Hansson M, Gulliksson H, Shanwell A. Storage of buffy-coat-derived platelets in additive solutions at 4 degrees $C$ and 22 degrees $C$ : flow cytometry analysis of platelet glycoprotein expression. Vox Sang 2007;93(01):27-36

11 Wood B, Padula MP, Marks DC, Johnson L. Refrigerated storage of platelets initiates changes in platelet surface marker expression and localization of intracellular proteins. Transfusion 2016;56 (10):2548-2559

12 Valeri CR. Circulation and hemostatic effectiveness of platelets stored at $4 \mathrm{C}$ or $22 \mathrm{C}$ : studies in aspirin-treated normal volunteers. Transfusion 1976;16(01):20-23

13 Becker GA, Tuccelli M, Kunicki T, Chalos MK, Aster RH. Studies of platelet concentrates stored at 22 C nad 4 C. Transfusion 1973;13 (02):61-68

14 Koerner K. Platelet function after shipment of room temperature platelet concentrates. Vox Sang 1983;44(01):37-41

15 Watts SE, Tunbridge LJ, Smith K, Lloyd JV. Storage of platelets for tests of platelet function: effects of temperature on platelet aggregation, platelet morphology and liberation of beta-thromboglobulin. Thromb Res 1986;44(03):365-376

16 Wallentin L, Becker RC, Budaj A, et al; PLATO Investigators. Ticagrelor versus clopidogrel in patients with acute coronary syndromes. N Engl J Med 2009;361(11):1045-1057

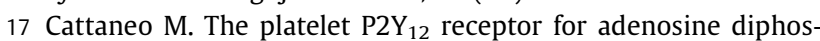
phate: congenital and drug-induced defects. Blood 2011;117(07): 2102-2112

18 Gachet C. P2 receptors, platelet function and pharmacological implications. Thromb Haemost 2008;99(03):466-472

19 Jin J, Daniel JL, Kunapuli SP. Molecular basis for ADP-induced platelet activation. II. The P2Y1 receptor mediates ADP-induced intracellular calcium mobilization and shape change in platelets. J Biol Chem 1998;273(04):2030-2034

20 Jin J, Kunapuli SP. Coactivation of two different $G$ protein-coupled receptors is essential for ADP-induced platelet aggregation. Proc Natl Acad Sci U S A 1998;95(14):8070-8074

21 Vial C, Rolf MG, Mahaut-Smith MP, Evans RJ. A study of P2 $\times 1$ receptor function in murine megakaryocytes and human platelets reveals synergy with P2Y receptors. Br J Pharmacol 2002;135(02): 363-372

22 Schwarz UR, Geiger J, Walter U, Eigenthaler M. Flow cytometry analysis of intracellularVASP phosphorylation for the assessment of activating and inhibitory signal transduction pathways in human platelets-definition and detection of ticlopidine/clopidogrel effects. Thromb Haemost 1999;82(03):1145-1152

23 Liu EC, Abell LM. Development and validation of a platelet calcium flux assay using a fluorescent imaging plate reader. Anal Biochem 2006;357(02):216-224

24 Jóhannsson F, Guðmundsson S, Paglia G, et al. Systems analysis of metabolism in platelet concentrates during storage in platelet additive solution. Biochem J 2018;475(13):2225-2240

25 Marini I, Aurich K, Jouni R, et al. Cold storage of platelets in additive solution: the impact of residual plasma in apheresis platelet concentrates. Haematologica 2019;104(01):207-214

26 Murphy S, Shimizu T, Miripol J. Platelet storage for transfusion in synthetic media: further optimization of ingredients and definition of their roles. Blood 1995;86(10):3951-3960

27 Johnson L, Tan S, Wood B, Davis A, Marks DC. Refrigeration and cryopreservation of platelets differentially affect platelet metab- olism and function: a comparison with conventional platelet storage conditions. Transfusion 2016;56(07):1807-1818

28 Reddoch KM, Pidcoke HF, Montgomery RK, et al. Hemostatic function of apheresis platelets stored at $4^{\circ} \mathrm{C}$ and $22^{\circ} \mathrm{C}$. Shock 2014;41(Suppl 1):54-61

29 Hoffmeister KM, Felbinger TW, Falet $\mathrm{H}$, et al. The clearance mechanism of chilled blood platelets. Cell 2003;112(01):87-97

30 Koessler J, Trulley VN, Bosch A, et al. The role of agonist-induced activation and inhibition for the regulation of purinergic receptor expression in human platelets. Thromb Res 2018;168:40-46

31 Oliver AE, Tablin F, Walker NJ, Crowe JH. The internal calcium concentration of human platelets increases during chilling. Biochim Biophys Acta 1999;1416(1-2):349-360

32 Koessler J, Weber K, Koessler A, Yilmaz P, Boeck M, Kobsar A. Expression and function of purinergic receptors in platelets from apheresis-derived platelet concentrates. Blood Transfus 2016;14 (06):545-551

33 Geiger J, Teichmann L, Grossmann R, et al. Monitoring of clopidogrel action: comparison of methods. Clin Chem 2005;51(06): 957-965

34 Kobsar A, Koessler J, Kehrer L, Gambaryan S, Walter U. The thrombin inhibitors hirudin and Refludan $\left({ }^{\circledR}\right)$ activate the soluble guanylyl cyclase and the cGMP pathway in washed human platelets. Thromb Haemost 2012;107(03):521-529

35 Schwarz UR, Walter U, Eigenthaler M. Taming platelets with cyclic nucleotides. Biochem Pharmacol 2001;62(09):1153-1161

36 Walter U, Eigenthaler M, Geiger J, Reinhard M. Role of cyclic nucleotide-dependent protein kinases and their common substrate VASP in the regulation of human platelets. Adv Exp Med Biol 1993;344:237-249

37 Biochemistry IUo. Committee MBN, NC-IUBMB, Webb EC, Stalmans W. Enzyme Nomenclature 1992: Recommendations of the Nomenclature Committee of the International Union of Biochemistry and Molecular Biology and the Nomenclature and Classification of Enzymes. San Diego, California, USA: Academic Press; 1992

38 Rene E, Pecker F, Stengel D, Hanoune J. Thermodependence of basal and stimulated rat liver adenylate cyclase. A re-evaluation. J Biol Chem 1978;253(03):838-841

39 Orly J, Schramm M. Fatty acids as modulators of membrane functions: catecholamine-activated adenylate cyclase of the turkey erythrocyte. Proc Natl Acad Sci U S A 1975;72(09):3433-3437

40 Scott WA. Adenosine $3^{\prime}: 5^{\prime}$-cyclic monophosphate deficiency in Neurospora crassa. Proc Natl Acad Sci US A 1976;73(09):2995-2999

41 Baude EJ, Arora VK, Yu S, Garbers DL, Wedel BJ. The cloning of a Caenorhabditis elegans guanylyl cyclase and the construction of a ligand-sensitive mammalian/nematode chimeric receptor. J Biol Chem 1997;272(25):16035-16039

42 Chao YC, Chen CC, Lin YC, Breer H, Fleischer J, Yang RB. Receptor guanylyl cyclase- $G$ is a novel thermosensory protein activated by cool temperatures. EMBO J 2015;34(03):294-306

43 Yu S, Avery L, Baude E, Garbers DL. Guanylyl cyclase expression in specific sensory neurons: a new family of chemosensory receptors. Proc Natl Acad Sci U S A 1997;94(07):3384-3387

44 Vassallo RR, Murphy S. A critical comparison of platelet preparation methods. Curr Opin Hematol 2006;13(05):323-330

45 Waters L, Cameron M, Padula MP, Marks DC, Johnson L. Refrigeration, cryopreservation and pathogen inactivation: an updated perspective on platelet storage conditions. Vox Sang 2018;113 (04):317-328

46 Hellstern P, Stürzebecher U, Wuchold B, et al. Preservation of in vitro function of platelets stored in the presence of a synthetic dual inhibitor of factor Xa and thrombin. J Thromb Haemost 2007; 5(10):2119-2126

47 Kaiser AF, Endres HG, Mügge A, Neubauer H. BAPA, a synthetic dual inhibitor of Factor $\mathrm{Xa}$ and Thrombin, extends the storagetime to a maximum of 12 hours in ADP- and 24 hours in arachidonic acid-induced impedance aggregometry. Scand J Clin Lab Invest 2011;71(03):253-256 